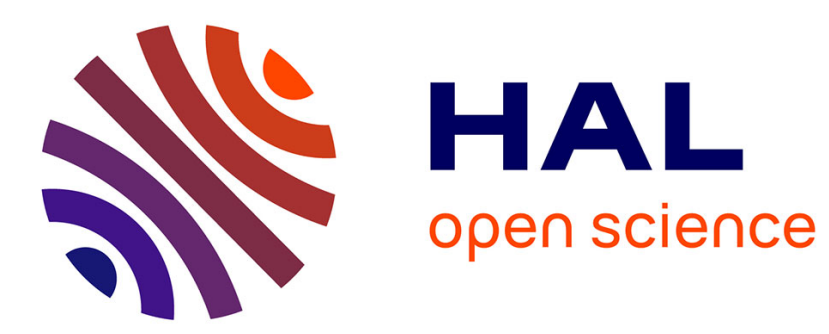

\title{
Influence of the elastic deformation of a foam on its mobility in channels of linearly varying width
} Benjamin Dollet, Sian A. Jones, Yves Méheust, Isabelle Cantat

\section{To cite this version:}

Benjamin Dollet, Sian A. Jones, Yves Méheust, Isabelle Cantat. Influence of the elastic deformation of a foam on its mobility in channels of linearly varying width. Physical Review E: Statistical, Nonlinear, and Soft Matter Physics, 2014, 90 (2), pp.art. nº23006. 10.1103/PhysRevE.90.023006 . insu-01068121

\section{HAL Id: insu-01068121 \\ https://hal-insu.archives-ouvertes.fr/insu-01068121}

Submitted on 25 Sep 2014

HAL is a multi-disciplinary open access archive for the deposit and dissemination of scientific research documents, whether they are published or not. The documents may come from teaching and research institutions in France or abroad, or from public or private research centers.
L'archive ouverte pluridisciplinaire HAL, est destinée au dépôt et à la diffusion de documents scientifiques de niveau recherche, publiés ou non, émanant des établissements d'enseignement et de recherche français ou étrangers, des laboratoires publics ou privés. 


\title{
Influence of the elastic deformation of a foam on its mobility in channels of linearly varying width
}

\author{
Benjamin Dollet, ${ }^{1}$ Siân A. Jones, ${ }^{1, *}$ Yves Méheust, ${ }^{2}$ and Isabelle Cantat ${ }^{1}$ \\ ${ }^{1}$ Institut de Physique de Rennes, Université Rennes 1, CNRS UMR No. 6251, Campus Beaulieu, 35042 Rennes Cedex, France \\ ${ }^{2}$ Géosciences, Université Rennes 1, CNRS UMR No. 6118, Campus Beaulieu, 35042 Rennes Cedex, France
}

(Received 20 December 2013; published 13 August 2014)

\begin{abstract}
We study foam flow in an elementary model porous medium consisting of a convergent and a divergent channel positioned side by side and possessing a fixed joint porosity. Configurations of converging or diverging channels are ubiquitous at the pore scale in porous media, as all channels linking pores possess a converging and diverging part. The resulting flow kinematics imposes asymmetric bubble deformations in the two channels, which modulate foam-wall friction and strongly impact the flux distribution. We measure, as well as quantitatively predict, the ratio of the fluxes in the two channels as a function of the channel widths by modeling pressure drops of both viscous and capillary origins. This study reveals the crucial importance of boundary-induced bubble deformation on the mobility of a flowing foam, resulting in particular in flow irreversibility.
\end{abstract}

DOI: 10.1103/PhysRevE.90.023006

PACS number(s): 47.56.+r, 47.57.Bc, 83.80.Iz

\section{INTRODUCTION}

Aqueous foams are used in many different applications, e.g., personal care or ore flotation [1,2]. In most of these applications the foam is flowing and understanding foam rheology is a fundamental challenge [3] relevant for the industry [2]. In particular, how an aqueous foam will flow though a porous material is important in soil remediation processes [4,5] and enhanced oil recovery [6,7]. The subtle role of the capillary forces in the complex and confined geometries characteristic of porous media is now well understood $[8,9]$. However, few studies have addressed nonquasistatic flows in such geometries [10-13] and a crucial pending issue is to understand the mobility of a foam through a disordered array of interconnected channels.

For highly confined foams forming trains of films (commonly known as bamboo foams), variations of pore section induce film curvature and hence jumps of Laplace pressure, which affect the pressure drop [14]. These capillary effects are dominant at low velocity and are complemented by viscous effects at higher velocity. Foams flowing along smooth wetting walls undergo a plug flow. The pressure drop then depends on the foam-wall friction coefficient [15], defined as the ratio between the tangential stress at the wall and the foam velocity. This coefficient is controlled by the sliding motion of menisci past the walls $[6,16,17]$. It depends on their total length per unit area, thus on bubble size, and on their orientation with respect to their velocity [18]. The latter dependence explains the peculiar pressure drops observed as ordered structures form when only few bubbles remain in the pore section [13,18-20]. In this paper we show that the mobility is also coupled to the elastic deformations, even if the foam remains disordered (thus not structured under confinement). Considering a convergent and a divergent channel with smooth wetting walls, which are configurations ubiquitous in porous media [21], and positioning them in parallel within a Hele-Shaw cell, we observe a flux difference for channels of identical average cross section and opening angle: The Stokes flow reversibility

\footnotetext{
*Present address: Faculty of Civil Engineering and Geosciences, Delft University of Technology, 2628CN Delft, The Netherlands.
}

[22] is thus lost. This cannot be explained by capillary effects; the coupling between the friction coefficient and elastic deformations is essential. We propose a model based on these two ingredients that fits the measurements of the flux ratio versus the section ratio between the two channels over two decades.

\section{MATERIALS AND METHODS}

The flow cell was realized by inserting a tilted obstacle in a Hele-Shaw cell (Fig. 1) of width $6 \mathrm{~cm}$ and gap $h=2 \mathrm{~mm}$. The obstacle could be moved spanwise so as to vary the widths $a_{\mathrm{c}}$ and $a_{\mathrm{d}}$ but keep their sum, hence the porosity of the medium, constant: $a_{\mathrm{c}}+a_{\mathrm{d}}=2 \mathrm{~cm}$. The foam was created by coflowing a solution of sodium dodecyl sulfate (SDS) in ultrapure water (concentration $10 \mathrm{~g} / \mathrm{L}$ ), which wetted the walls perfectly, and nitrogen through a T-junction far upstream the obstacle. This generated an assembly of bubbles of mean area $A$ big enough to form a monolayer spanning the cell gap, ensuring an easy visualization. The fluxes $Q_{\mathrm{c}}$ and $Q_{\mathrm{d}}$ in the convergent and divergent channels, respectively, were thus measured by image analysis. The liquid fraction was between $1 \%$ and $2 \%$ and the total foam flux $Q=Q_{\mathrm{c}}+Q_{\mathrm{d}}=0.68 \mathrm{~cm}^{3} / \mathrm{s}$ was kept constant within $3 \%$, unless explicitly stated otherwise. The experiments were carried out for a width $a_{\mathrm{c}}$ (at the exit of the convergent channel) ranging between 0.2 and $1.8 \mathrm{~cm}$, each for two values of $A: 0.11 \pm 0.02$ and $0.45 \pm 0.10 \mathrm{~cm}^{2}$.

Typical foam structures are shown in Fig. 1. The bubbles are elongated streamwise (spanwise) in the convergent (divergent) channel.

\section{SYMMETRIC CONFIGURATION}

We first focus on the symmetric case $a_{\mathrm{c}}=a_{\mathrm{d}}$ [Fig. 1(b)]. We measure $Q_{\mathrm{d}} / Q_{\mathrm{c}}=0.69$ for $A=0.45 \mathrm{~cm}^{2}$ and $Q_{\mathrm{d}} / Q_{\mathrm{c}}=0.67$ for $A=0.11 \mathrm{~cm}^{2}$, values significantly lower than one, even though the average cross section is equal in both channels. We have measured no significant change in this flux ratio as the total flux was varied between 0.11 and $1.4 \mathrm{~cm}^{3} / \mathrm{s}$ (data not shown). As a qualitative explanation, the friction on a soap film sliding along a wall increases with its spanwise projected length [18]; hence bubbles have a higher friction in 
(a)

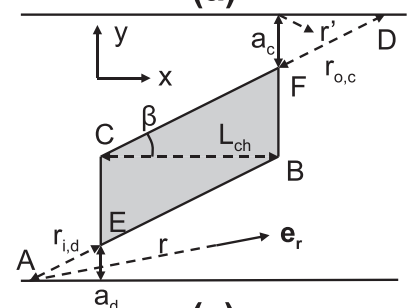

(c)
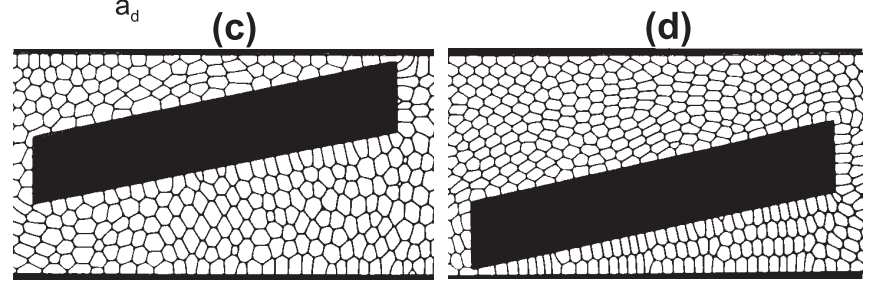

FIG. 1. (a) Sketch of the flow configuration (not to scale). The total width is $6 \mathrm{~cm}$. The obstacle dimensions are $L_{\mathrm{ch}}=10 \mathrm{~cm}, C E$ and $B F=2 \mathrm{~cm}$, and $\beta=11.3^{\circ}$. The lengths $r_{\mathrm{o}, \mathrm{d}}$ and $r_{\mathrm{i}, \mathrm{c}}$ used in the text are the distances $A B$ and $C D$, respectively. Also shown are binary images of foam flowing through the system for (b) $a_{\mathrm{c}}=1.0 \mathrm{~cm}$, (c) $a_{\mathrm{c}}=0.2 \mathrm{~cm}$, and (d) $a_{\mathrm{c}}=1.8 \mathrm{~cm}$. Flow is from left to right.

the divergent channel than in the convergent one. Considering capillary effects, we will show later that they cancel out in this symmetric case.

To quantify the viscous pressure drop across a channel, we recall that for soap solutions with negligible interfacial viscoelasticity such as SDS, a planar film of end-to-end vector $\mathbf{L}$ and length $L=|\mathbf{L}|$, sliding at a velocity $\mathbf{v}$ along a wall and of unit normal vector $\hat{\mathbf{n}}$ such that $\mathbf{v} \cdot \hat{\mathbf{n}}>0$, experiences a friction force $[18,23] \mathbf{F}=-\lambda \gamma \mathrm{Ca}^{2 / 3} L \hat{\mathbf{n}}$, where $\mathrm{Ca}=\eta|\mathbf{v} \cdot \hat{\mathbf{n}}| / \gamma$ is the capillary number, $\lambda$ a dimensionless prefactor that equals $37 \pm 3$ in our range of liquid fraction [13], $\gamma=36.8 \mathrm{mN} / \mathrm{m}$ the surface tension, and $\eta=10^{-3} \mathrm{~Pa}$ s the bulk viscosity. For negligible elastic stress, the pressure gradient $\nabla P_{\mathrm{vis}}$ is of purely viscous origin. A force balance shows that it equals $2 \sigma_{w} / h$, $\sigma_{w}$ being the tangential viscous stress at the wall. The factor 2 comes from the top and bottom plates. The foam mobility in the channel is characterized by the friction coefficient $\xi$ defined by $\sigma_{w}=-\xi \mathbf{v}$. At the scale of a box of area $A_{\text {box }}$ and for a low liquid fraction $\boldsymbol{\sigma}_{w}=\left(N / A_{\text {box }}\right)\langle|\mathbf{F} \cdot \hat{\mathbf{v}}|\rangle \hat{\mathbf{v}}$, where $N$ is the average number of films in the box, $\hat{\mathbf{v}}=\mathbf{v} / v$ and $\langle\cdot\rangle$ denotes the average taken over all the films in the box. Since the flow is steady, $\sigma_{w}$ is time averaged to optimize statistics. At the scale of a bubble of area $A$, which has on average six edges, each shared with one neighbor, one obtains

$$
\xi=\frac{3}{A} \lambda \gamma^{1 / 3} \eta^{2 / 3} \mathcal{L} v^{-1 / 3},
$$

where $\mathcal{L}=\left\langle|\hat{\mathbf{v}} \cdot \hat{\mathbf{n}}|^{5 / 3} L\right\rangle$. The value of $\mathcal{L}$ depends on the elastic deformation, which can be quantified by various tensors such as $\tilde{\boldsymbol{\sigma}}=\langle\mathbf{L} \otimes \mathbf{L} / L\rangle$, which, up to a prefactor proportional to surface tension, is the elastic stress in the foam [24].

We plot in Fig. 2 the velocity field together with tensor $\tilde{\boldsymbol{\sigma}}$, which is shown as ellipses whose major (minor) axis represents the magnitude of elongation (compression). We also plot in Fig. 3 the evolution along both channels of the streamwise component of the velocity $v_{x}$, of $\mathcal{L}$, and of the viscous pressure
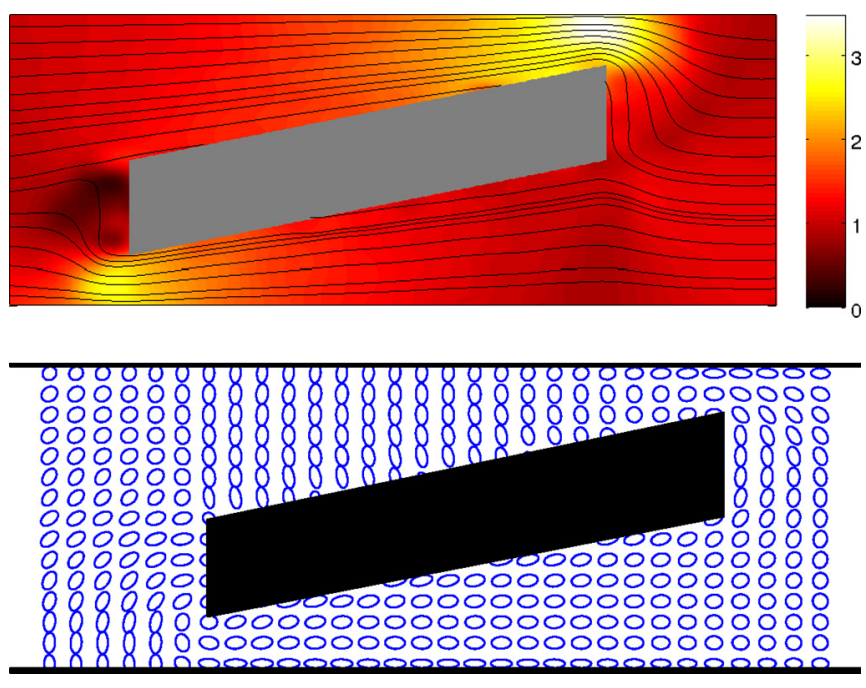

FIG. 2. (Color online) Maps of the velocity normalized by the average inlet velocity (top) and of the deformation quantified by the tensor $\tilde{\boldsymbol{\sigma}}$ (bottom), in the symmetric case $a_{\mathrm{c}}=a_{\mathrm{d}}$.

drop deduced from (1) using the experimentally measured values of $\mathcal{L}$ and $\mathbf{v}$. The velocity increases along the convergent channel and drops abruptly at the exit. The opposite variation occurs in the divergent channel, as expected. In the convergent channel, the length $\mathcal{L}$ quickly reaches a plateau, corresponding to the yield point, and increases sharply at the exit, like at a constriction exit [25]. In the divergent channel, it tends towards a higher plateau value, after an increase as the elongation direction flips from streamwise at the entrance to spanwise. Most interestingly, the overall viscous pressure drop along the two channels is the same within 5\%, as expected for channels in parallel. This finally proves that the foam flux can differ in two channels that are identical but oriented with $180^{\circ}$
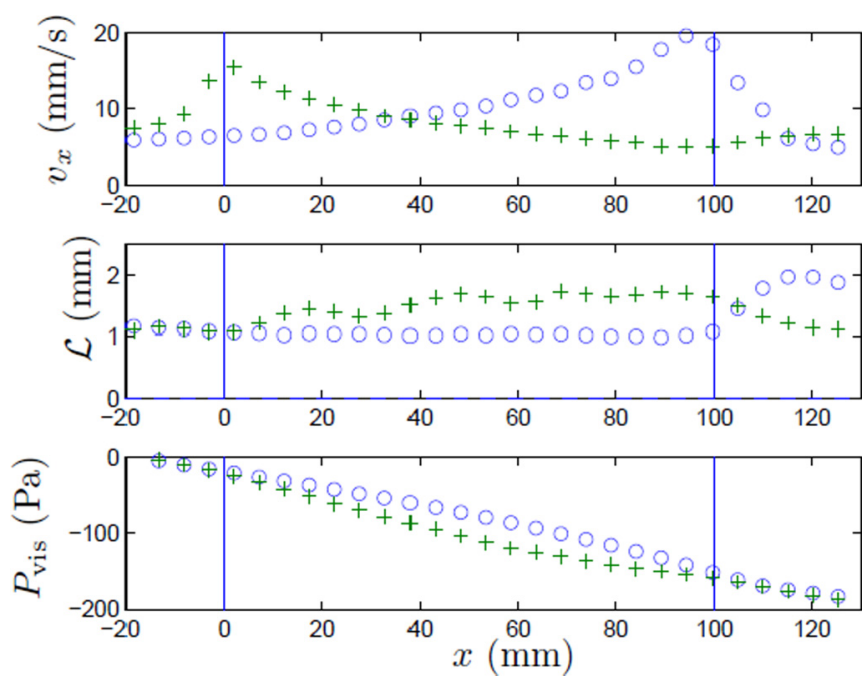

FIG. 3. (Color online) Shown, from top to bottom, are the velocity component $v_{x}$, length $\mathcal{L}$, and pressure (set to zero at $x=-19 \mathrm{~mm}$ ) as functions of $x$ along the convergent [ $\circ$; dash-dotted line in Fig. 1(b)] and divergent $[+;$ dotted line in Fig. 1(b)] channels. The origin of the $x$ axis is taken at the entrance of the two channels. Vertical lines indicate the locations of the entrance and exit of the two channels. 
rotational symmetry to each other, if the boundary-induced flow configuration enforces an elastic deformation that impacts the friction coefficient. More generally, the foam flow along a channel of variable width is not necessarily reversible.

\section{ASYMMETRIC CONFIGURATIONS}

We now investigate asymmetric configurations at arbitrary $a_{\mathrm{c}} / a_{\mathrm{d}}$. We measure the flux ratio $Q_{\mathrm{d}} / Q_{\mathrm{c}}$ over two decades of $a_{\mathrm{d}} / a_{\mathrm{c}}$ (Fig. 4). It increases strongly with the width ratio. For the two tested bubble sizes, the flux ratio was found to be insensitive to the bubble size for $a_{\mathrm{d}} / a_{\mathrm{c}} \geqslant 1$. On the other hand, for $a_{\mathrm{d}}<a_{\mathrm{c}}$, it depends on the bubble size: It is larger for smaller bubbles.

We now predict $Q_{\mathrm{d}} / Q_{\mathrm{c}}$ versus $a_{\mathrm{d}} / a_{\mathrm{c}}$, first assuming an equal viscous pressure drop along the two channels, which was just shown to be valid in the symmetric case. We first relate the flux to the velocity field in the divergent channel. The pressure drop is about $10^{2} \mathrm{~Pa}$ (Fig. 3), much lower than the inverse of the bulk modulus of a foam, of the order of the atmospheric pressure $\left(10^{5} \mathrm{~Pa}\right)$ since foam mostly consists of gas. Hence, the flow is incompressible and the velocity obeys the continuity equation $\boldsymbol{\nabla} \cdot \mathbf{v}=0$. Furthermore, the foam slips perfectly along the smooth side walls in our range of control parameters (Fig. 2). Hence, neglecting entrance and exit effects, there is a polar symmetry in the divergent channel $\mathbf{v}=v(r) \mathbf{e}_{r}$. We have checked in the symmetric case that polar symmetry holds within $2 \%$. The continuity equation then yields

$$
\mathbf{v}=\frac{Q_{\mathrm{d}}}{h \beta r} \mathbf{e}_{r}
$$

where $\beta$ is the tilt angle (Fig. 1).

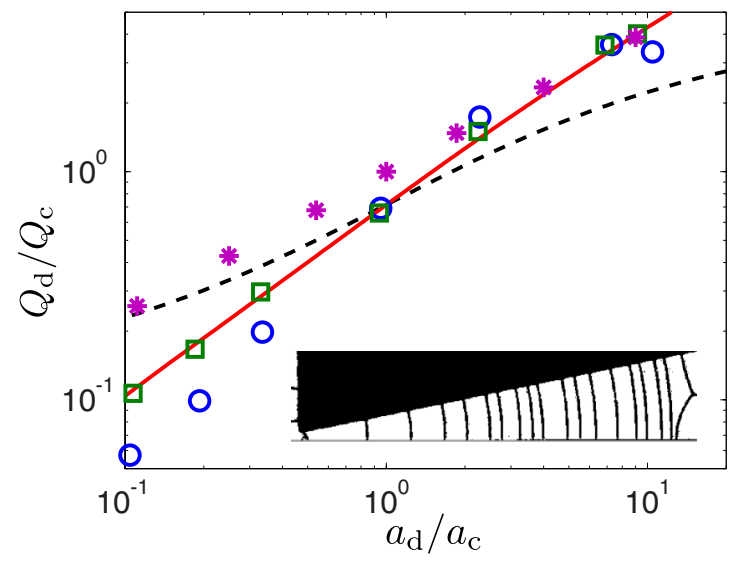

FIG. 4. (Color online) Flux ratio $Q_{\mathrm{d}} / Q_{\mathrm{c}}$ plotted versus $a_{\mathrm{d}} / a_{\mathrm{c}}$. The experimental data for two different bubble areas are $0.11 \pm$ $0.02 \mathrm{~cm}^{2}(\square)$ and $0.45 \pm 0.10 \mathrm{~cm}^{2}(\circ)$. The dashed curve is from Eq. (5) with $\mathcal{L}_{\mathrm{c}}^{*} / \mathcal{L}_{\mathrm{d}}^{*}=0.80$. The solid curve is from Eq. (7) with $\mathcal{L}_{\mathrm{c}}^{*} / \mathcal{L}_{\mathrm{d}}^{*}=0.80$ and $B=8.7$. The asterisks denote results from $3 \mathrm{D}$ simulations of a Stokes flow (Newtonian fluid, vanishing Reynolds number). The inset shows a zoom at the entrance of the divergent channel when it is narrow and the bubbles are big, showing the structure as a bamboo foam.
From (1), the pressure drop along the divergent channel $\Delta P_{\mathrm{vis}, \mathrm{d}}=\int_{r_{\mathrm{i}, \mathrm{d}}}^{r_{\mathrm{od}}} \nabla P_{\mathrm{vis}} \cdot \mathbf{e}_{r} d r$ equals

$$
\Delta P_{\mathrm{vis}, \mathrm{d}}=-\frac{6 \lambda \gamma^{1 / 3} \eta^{2 / 3}}{h A} \int_{r_{\mathrm{i}, \mathrm{d}}}^{r_{\mathrm{o}, \mathrm{d}}} \mathcal{L}_{\mathrm{d}}(r) v(r)^{2 / 3} d r
$$

$\left(r_{\mathrm{i}, \mathrm{d}}\right.$ and $r_{\mathrm{O}, \mathrm{d}}$ are defined in Fig. 1). We define the $v^{2 / 3}$-weighted average of $\mathcal{L}_{\mathrm{d}}, \mathcal{L}_{\mathrm{d}}^{*}=\int_{r_{\mathrm{i}, \mathrm{d}}}^{r_{\mathrm{o}}} \mathcal{L}_{\mathrm{d}}(r) v(r)^{2 / 3} d r / \int_{r_{\mathrm{i}, \mathrm{d}}}^{r_{\mathrm{o}}} v(r)^{2 / 3} d r$, and using (2) we obtain

$$
\Delta P_{\mathrm{vis}, \mathrm{d}}=-C \mathcal{L}_{\mathrm{d}}^{*} Q_{\mathrm{d}}^{2 / 3}\left(r_{\mathrm{o}, \mathrm{d}}^{1 / 3}-r_{\mathrm{i}, \mathrm{d}}^{1 / 3}\right),
$$

where $C=18 \lambda \gamma^{1 / 3} \eta^{2 / 3} / h^{5 / 3} \beta^{2 / 3} A$. Similarly, the pressure drop in the convergent channel is $\Delta P_{\text {vis }, \mathrm{c}}=-C \mathcal{L}_{\mathrm{c}}^{*} Q_{\mathrm{c}}^{2 / 3}\left(r_{\mathrm{i}, \mathrm{c}}^{1 / 3}-\right.$ $\left.r_{\mathrm{o}, \mathrm{c}}^{1 / 3}\right)$. The equality between pressure drops $\Delta P_{\mathrm{vis}, \mathrm{c}}=\Delta P_{\mathrm{vis}, \mathrm{d}}$ finally yields a prediction for the flux ratio

$$
\frac{Q_{\mathrm{d}}}{Q_{\mathrm{c}}}=\left(\frac{r_{\mathrm{i}, \mathrm{c}}^{1 / 3}-r_{\mathrm{o}, \mathrm{c}}^{1 / 3}}{r_{\mathrm{o}, \mathrm{d}}^{1 / 3}-r_{\mathrm{i}, \mathrm{d}}^{1 / 3}}\right)^{3 / 2}\left(\frac{\mathcal{L}_{\mathrm{c}}^{*}}{\mathcal{L}_{\mathrm{d}}^{*}}\right)^{3 / 2} .
$$

We compute from the experiments for the symmetric case $\mathcal{L}_{\mathrm{d}}^{*} / \mathcal{L}_{\mathrm{c}}^{*}=0.80$, which we keep for all cases, as the yield value for $\mathcal{L}$ is reached at a short distance from the inlet in both channels and has been measured to be independent of the channel width. Using $\mathcal{L}_{\mathrm{d}}^{*} / \mathcal{L}_{\mathrm{c}}^{*}=0.80$ in (5) provides a prediction plotted as a dashed line in Fig. 4. Prediction and measurements agree on the symmetric case, but the flux ratio is overpredicted (underpredicted) at low (high) $a_{\mathrm{c}} / a_{\mathrm{d}}$.

We now consider the hitherto disregarded capillary effects. These are shown best in Fig. 1(c), where the films exiting the convergent channel are circular arcs. The capillary pressure jump across a soap film of curvature $\kappa$ equals $2 \gamma \kappa$ from the concave side to the convex one. Hence, there is a capillary pressure drop at the exit of the convergent channel, in addition to the viscous pressure drop [14]. Moreover, even when films are not as curved, this capillary effect extends up to the separation point of the flow. Such a capillary effect is also seen in the divergent channel, most clearly for a bamboo foam: In this case, the films bulge towards the exit [Fig. 1(d)]. Interestingly, there is no clear evidence of such capillary effects in the other regions of the flow.

Given the relatively good radial symmetry of the foam at the exit of the convergent channel (Fig. 2), we model the capillary pressure drop by simply assuming that the average film curvature scales as $1 / r^{\prime}$ for $r^{\prime}$ between $a_{\mathrm{c}}$ (the narrowest width at the exit) and $a_{\mathrm{c}}+F B$ [at the separation point; see Fig. 1(a)]. This scaling is exact for a bamboo foam and captures the trend qualitatively in less confined configurations. Since the density of films per unit length is of order $1 / \sqrt{A}$, the capillary pressure drop at the exit of the convergent channel is $\Delta P_{\text {cap, }}=$ $-B_{\mathrm{c}} \int_{a_{\mathrm{c}}}^{a_{\mathrm{c}}+F B}\left(\gamma / r^{\prime}\right)\left(d r^{\prime} / \sqrt{A}\right)$, with a positive dimensionless prefactor $B_{\mathrm{c}}$ of order 1 . Since $1+F B / a_{\mathrm{c}}=r_{\mathrm{i}, \mathrm{c}} / r_{\mathrm{o}, \mathrm{c}}$, we get

$$
\Delta P_{\text {cap }, \mathrm{c}}=-B_{\mathrm{c}} \frac{\gamma}{\sqrt{A}} \ln \frac{r_{\mathrm{i}, \mathrm{c}}}{r_{\mathrm{o}, \mathrm{c}}} .
$$

Similarly, in the divergent channel, the capillary pressure drop is $\Delta P_{\text {cap }, \mathrm{d}}=-B_{\mathrm{d}}(\gamma / \sqrt{A}) \ln \left(r_{\mathrm{o}, \mathrm{d}} / r_{\mathrm{i}, \mathrm{d}}\right)$.

Accounting for this capillary contribution, the flux ratio is now determined by $\Delta P_{\mathrm{vis}, \mathrm{c}}+\Delta P_{\text {cap }, \mathrm{c}}=\Delta P_{\mathrm{vis}, \mathrm{d}}+\Delta P_{\text {cap }, \mathrm{d}}$. We assume that $B_{\mathrm{c}}=B_{\mathrm{d}}=B$ for simplicity. After (4), (6), 
and similar expressions for $\Delta P_{\mathrm{vis}, \mathrm{c}}$ and $\Delta P_{\text {cap }, \mathrm{d}}$, the flux ratio obeys

$$
\begin{aligned}
& \frac{1}{\left(1+Q_{\mathrm{d}} / Q_{\mathrm{c}}\right)^{2 / 3}} \mathcal{L}_{\mathrm{c}}^{*}\left(r_{\mathrm{i}, \mathrm{c}}^{1 / 3}-r_{\mathrm{o}, \mathrm{c}}^{1 / 3}\right)+\bar{B} \ln \frac{r_{\mathrm{i}, \mathrm{c}}}{r_{\mathrm{o}, \mathrm{c}}} \\
& =\left(\frac{Q_{\mathrm{d}} / Q_{\mathrm{c}}}{1+Q_{\mathrm{d}} / Q_{\mathrm{c}}}\right)^{2 / 3} \mathcal{L}_{\mathrm{d}}^{*}\left(r_{\mathrm{o}, \mathrm{d}}^{1 / 3}-r_{\mathrm{i}, \mathrm{d}}^{1 / 3}\right)+\bar{B} \ln \frac{r_{\mathrm{i}, \mathrm{d}}}{r_{\mathrm{o}, \mathrm{d}}}
\end{aligned}
$$

with $\bar{B}=B \gamma / C Q^{2 / 3} A^{1 / 2}$. Since the dependence of $B$ on bubble geometry is unknown, we solve this equation numerically with $B$ as a free parameter. Taking $B=8.7$ yields the solid line in Fig. 4, in excellent agreement with all data at small bubble size. We can therefore conclude that the flux ratio can be predicted by accounting for both the influence of bubble orientation on dissipation and capillary effects.

As a side remark, the capillary effects in the divergent channel and at the exit of the convergent one cancel out in (7) precisely for $a_{\mathrm{c}}=a_{\mathrm{d}}$. Hence, although our estimate of the viscous pressure drop (Fig. 3) is only a partial estimation of the full pressure drop, it was relevant a posteriori to discuss the symmetric case in terms of a viscous pressure drop only.

At large bubble size, the flux ratio remains overestimated at small $a_{\mathrm{d}} / a_{\mathrm{c}}$. In such cases, the foam is highly confined at the entrance of the divergent channel, leading to a succession of ordered bamboo and staircase structures [Fig. 1(d) and inset of Fig. 4], which has already been reported in [26]. These structures build up until they become unstable and transit, e.g., from bamboo to a single staircase [19,20,27,28]. Just before a transition, bubbles are extremely elongated spanwise, much more than in regions of unconfined foam. This drastically increases friction and explains why the flux drops significantly in the narrowest divergent channels and why the flux ratio drops more for larger bubbles (Fig. 4), because then at given channel width, confinement effects are stronger. We do not attempt to model this effect because the ordering of a confined foam exists only in a certain range of ratio of the width channel to the bubble size, whose dependence on the foam polydispersity is unknown. Hence, the model based on (7) does not apply when $a_{\mathrm{d}}$ is of the order of the bubble size or smaller.

For comparison, we have simulated the three-dimensional (3D) flow of a Newtonian fluid at vanishing Reynolds number (Stokes flow) in the same geometry, using the FREEFEM++ finite-element partial differential equation solver [29] (see the Appendix for details). The flux ratio increases less versus $a_{\mathrm{d}} / a_{\mathrm{c}}$ for a Newtonian fluid than for a foam (Fig. 4), suggesting that the latter invades narrow divergent pores less efficiently than Newtonian fluids. This is in marked contrast to the case of two parallel straight channels, where foams were shown to invade narrow channels more efficiently than Newtonian fluids [13]. In a straight channel, foam-wall friction depends on the width only when the latter is of the order of the bubble size or smaller, as the foam becomes highly ordered in structures such as bamboo or staircases. On the other hand, in convergent or divergent channels, friction depends on the elastic deformation induced by the geometrical boundary conditions even if foam is unconfined. This illustrates the complex interplay between structure, capillary, and viscous effects in foam flows through porous media.

\section{CONCLUSION}

We have shown that the structure of a flowing foam is greatly impacted by the boundary-induced flow profile, even if the foam is not highly confined laterally. This both modulates the foam-wall friction coefficient and creates a significant capillary contribution to the pressure drop. Quantifying these two effects, we have predicted the flux ratio between the two channels versus their width ratio. This coupling between the flow kinematics, the foam deformation, and the resulting pressure drop is expected to occur in porous media as pores narrow or widen, resulting in particular in a fundamental irreversibility of foam flows. In this respect, for the symmetric ( $a_{\mathrm{d}}=a_{\mathrm{c}}$ ) configuration, the flux ratio is the inverse of the effective viscosity ratio [11], hence foam exhibits different effective viscosities in the two channels. The resulting irreversibility of the flow, though investigated here on the scale of a single channel, could explain phenomena of flow irreversibility and hysteresis on the scale of the entire porous medium, as all porous media consist of pores linked by channels of nonuniform width. Our predictions are thus an important step toward the understanding of foam mobility in porous media. Future perspectives include extension to geometries consisting of cylindrical solid grains as well as to $3 \mathrm{D}$ porous media and more complex boundary conditions on the solid surfaces, including rough or nonwetting walls. Finally, the link between deformation and mobility for a foam is reminiscent of the structure-viscosity coupling existing in other elastic fluids, which is responsible, e.g., for the large elongational viscosity of polymers [30].

\section{ACKNOWLEDGMENT}

We acknowledge financial support from Région Bretagne (CREATE MOUSPORE).

\section{APPENDIX: NUMERICAL SIMULATION OF STATIONARY STOKES FLOWS IN THE EXPERIMENTAL GEOMETRY}

The flow of an incompressible Newtonian fluid at vanishingly low Reynolds number inside the experimental geometry was simulated numerically using a finite-element method. The equations to be solved are the continuity equation and the Stokes equation

$$
\begin{aligned}
& \boldsymbol{\nabla} \cdot \boldsymbol{v}=0, \\
& \nabla p^{\star}=\eta \boldsymbol{\Delta} \boldsymbol{v},
\end{aligned}
$$

where $v$ is the fluid velocity field, $\eta$ is its viscosity, and $p^{\star}=p+\rho g z$ is the pressure $p$ corrected for its hydrostatic component, $\rho$ being the fluid's volumetric mass density and $g$ the amplitude of the gravitational acceleration. The HeleShaw geometry was positioned horizontally and the boundary conditions were chosen to be (i) a null fluid velocity at all solid walls, including those of the central obstacle, and (ii) two iso- $p^{\star}$ surfaces as inlet- and outlet-vertical surfaces. Since we were interested in the flow distribution between the two channels on either side of the obstacle, the fluid viscosity and global drop in $p^{\star}$ could be chosen to be 1 without any loss of generality. 
The weak formulation for the stationary Stokes problem (A1) was derived as

$$
\begin{aligned}
& \iiint_{\mathcal{V}}\left[\sum_{i}\left(\nabla \psi_{i} \cdot \nabla v_{i}\right)-p^{\star}(\nabla \cdot \boldsymbol{\psi})-q(\nabla \cdot \boldsymbol{v})\right] d \mathcal{V} \\
& \quad+\oiint_{\Sigma}\left[p^{\star} \boldsymbol{\psi}+\sum_{i} \psi_{i} \nabla v_{i}\right] \cdot \hat{\boldsymbol{n}} d S=0,
\end{aligned}
$$

where $\Sigma$ is the closed solid boundary, $\mathcal{V}$ the volume included in $\Sigma$, and $\hat{\boldsymbol{n}}$ the unit vector normal to $\Sigma$ and oriented towards the outside. This formulation means that a weak solution $\left(p^{\star}, \boldsymbol{v}\right)$ was found as consisting of the functions that minimized the left-hand side of Eq. (A2) for all test functions $(q, \psi)$ belonging to the proper test function space and vanishing at the solid boundary $\Sigma$. The definition of the finite-element problem and the resulting minimization were implemented using the FREEFEM++ partial differential equation solver [31]. The test functions and finite elements to be used were chosen by the software.

The mesh was built vertically from a 2D mesh of a horizontal cut of the 3D geometry, after the two-dimensional mesh had been refined according to the gradient of the velocity field obtained as the solution of the Stokes flow on the initial homogeneous 2D mesh. The final 3D mesh was isotropic and more refined close to the solid boundaries; it contained about 50000 vertices. Note that to ensure an optimal balance between mesh resolution and number of vertices, we have chosen to define the length of the computational domain to twice the length of the obstacle $\left(L=2 L_{\mathrm{ch}}\right)$. Tests performed in

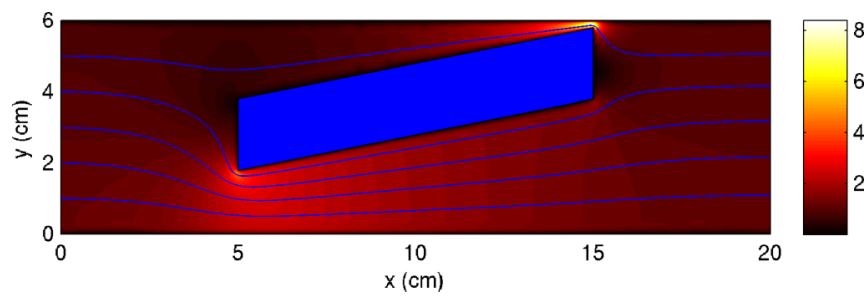

FIG. 5. (Color online) Map of the normalized local fluxes $\|\boldsymbol{q}(x, y)\| /\langle q\rangle$ for the geometry such that $a_{2}=9 a_{1}$, with the obstacle and a few streamlines drawn in blue.

the most asymmetric geometry $\left(a_{\mathrm{d}} / a_{\mathrm{c}}=0.11\right)$ with $L=4 L_{\mathrm{ch}}$ have shown that this choice does not impact the resulting $Q_{\mathrm{d}} / Q_{\mathrm{c}}$ values by more than about $1 \%$.

The solution exhibited a maximum relative fluctuation of the volumetric flow rate along the Hele-Shaw cell, as well as along the two separate channels, of a few percent. The volumetric flow rates averaged along the two channels were used to compute the dependence of $Q_{2} / Q_{1}$ on $a_{2} / a_{1}$ presented in this paper. A map of the local flux amplitude $\|\boldsymbol{q}(x, y)\|$ normalized by its average value $\langle q\rangle$ is shown in Fig. 5 for the most asymmetric investigated geometry. The local flux $\boldsymbol{q}$ is defined as the integral over the thickness of the Hele-Shaw cell of the horizontal component of the fluid velocity. The superimposed flow lines show that at the side boundaries of the computational box the flow still senses the presence of the obstacle to some extent; however, as mentioned above, the calculated $Q_{\mathrm{d}} / Q_{\mathrm{c}}$ values are hardly affected by this choice of computational box length $L$.
[1] I. Cantat, S. Cohen-Addad, F. Elias, F. Graner, R. Höhler, O. Pitois, F. Rouyer, and A. Saint-Jalmes, in Foams: Structure and Dynamics, edited by S. J. Cox (Oxford University Press, Oxford, 2013).

[2] Foam Engineering: Fundamentals and Applications, edited by P. Stevenson (Wiley, New York, 2012).

[3] S. Cohen-Addad, R. Höhler, and O. Pitois, Annu. Rev. Fluid Mech. 45, 241 (2013).

[4] C. N. Mulligan, R. N. Yong, and B. F. Gibbs, Eng. Geol. 60, 371 (2001).

[5] S. Wang and C. N. Mulligan, Chemosphere 57, 1079 (2004).

[6] G. J. Hirasaki and J. B. Lawson, Soc. Petrol. Eng. J. 25, 176 (1985).

[7] W. R. Rossen, Foam in Enhanced Oil Recovery (Dekker, Cleveland, 1996), pp. 413-465.

[8] W. R. Rossen, J. Colloid Interface Sci. 136, 1 (1990).

[9] S. J. Cox, S. Neethling, W. R. Rossen, W. Schleifenbaum, P. Schmidt-Wellenburg, and J. J. Cilliers, Colloids Surf. A 245, 143 (2004).

[10] K. G. Kornev, A. V. Neimark, and A. N. Rozhkov, Adv. Colloid Interface Sci. 82, 127 (1999).

[11] A. R. Kovscek and H. J. Bertin, Transp. Porous Media 52, 17 (2003).

[12] D. Du, P. Zitha, and F. Vermolen, Transp. Porous Media 86, 461 (2011).
[13] S. A. Jones, B. Dollet, Y. Méheust, S. J. Cox, and I. Cantat, Phys. Fluids 25, 063101 (2013).

[14] Q. Xu and W. R. Rossen, Colloids Surf. A 216, 175 (2003).

[15] L. Bocquet and E. Charlaix, Chem. Soc. Rev. 39, 1073 (2010).

[16] C. Raufaste, A. Foulon, and B. Dollet, Phys. Fluids 21, 053102 (2009).

[17] N. D. Denkov, S. Tcholakova, K. Golemanov, K. P. Ananthapadmanabhan, and A. Lips, Soft Matter 5, 3389 (2009).

[18] I. Cantat, N. Kern, and R. Delannay, Europhys. Lett. 65, 726 (2004).

[19] J. P. Raven and P. Marmottant, Phys. Rev. Lett. 97, 154501 (2006).

[20] J. P. Raven and P. Marmottant, Phys. Rev. Lett. 102, 084501 (2009).

[21] M. J. Blunt, B. Bijeljic, H. Dong, O. Gharbi, S. Iglauer, P. Mostaghimi, A. Paluszny, and C. Pentland, Adv. Water Resour. 51, 197 (2013).

[22] G. K. Batchelor, An Introduction to Fluid Dynamics (Cambridge University Press, Cambridge, 1967).

[23] N. Kern, D. Weaire, A. Martin, S. Hutzler, and S. J. Cox, Phys. Rev. E 70, 041411 (2004).

[24] G. K. Batchelor, J. Fluid Mech. 41, 545 (1970).

[25] B. Dollet, J. Rheol. 54, 741 (2010).

[26] W. Drenckhan, S. J. Cox, G. Delaney, H. Holste, D. Weaire, and N. Kern, Colloids Surf. A 263, 52 (2005). 
[27] M. A. Fortes, M. E. Rosa, M. F. Vaz, and P. I. C. Teixeira, Eur. Phys. J. E 15, 395 (2004).

[28] M. F. Vaz and S. J. Cox, Philos. Mag. Lett. 85, 415 (2005).

[29] F. Hecht, J. Numer. Math. 20, 251 (2012).
[30] R. G. Larson, The Structure and Rheology of Complex Fluids (Oxford University Press, Oxford, 1999).

[31] http://www.freefem.org/ff++. 